\title{
NOX4 Deletion in Male Mice Exacerbates the Effect of Ethanol on Trabecular Bone and Osteoblastogenesis ${ }^{[\$}$
}

\author{
James Watt, Alexander W. Alund, ${ }^{1}$ Casey F. Pulliam, Kelly E. Mercer, Larry J. Suva, \\ Jin-Ran Chen, and Martin J. J. Ronis
}

Department of Pharmacology and Experimental Therapeutics, Louisiana State University Health Sciences Center, New Orleans, Louisiana (J.W., C.F.P., M.J.J.R.); Interdisciplinary Biological Sciences Program (A.W.A.) and Department of Pediatrics, Arkansas Children's Nutrition Center (K.E.M., J.-R.C.), University of Arkansas for Medical Sciences, Little Rock, Arkansas; and Department of Veterinary Physiology and Pharmacology, Texas A\&M University, College Station, Texas (L.J.S.)

Received December 15, 2017; accepted March 21, 2018

\begin{abstract}
Chronic alcohol consumption increases bone resorption and decreases bone formation. A major component of ethanol (EtOH) pathology in bone is the generation of excess reactive oxygen species (ROS). The ROS-generating NADPH oxidase-4 (NOX4) is proposed to drive much of the $\mathrm{EtOH}$-induced suppression of bone formation. Here, 13-week-old male wild-type (WT) and NOX $4^{-1-}$ mice were pair fed (PF) a high-fat (35\%), Lieber-DeCarli liquid diet with or without $\mathrm{EtOH}$ at $30 \%$ of their total calories for 12 weeks. Micro-computed tomography analysis demonstrated significant decreases in trabecular bone volume/total volume (BV/TV) percentage and cortical thickness in WT, EtOH-fed mice compared with PF controls. EtOH-fed $\mathrm{NOX}^{-1-}$ mice also displayed decreased trabecular BV/TV and trabecular number compared with PF $(P<0.05)$. However, NOX4 ${ }^{-1-}$ mice were protected against $\mathrm{EtOH}$-induced decreases in cortical thickness
\end{abstract}

$(P<0.05)$ and decreases in collagen 1 and osteocalcin mRNA expression in cortical bone $(P<0.05)$. In WT and NOX4 ${ }^{-1-}$ vertebral bone, EtOH suppressed expression of Wnt signaling components that promote osteoblast maturation. A role for NOX4 in EtOH inhibition of osteoblast differentiation was further demonstrated by protection against $\mathrm{EtOH}$ inhibition of osteoblastogenesis in ex vivo bone marrow cultures from $\mathrm{NOX}^{-1-}$, but not $\mathrm{p} 47^{\text {phox- }-}$ mice lacking active NADPH oxidase-2. However, bone marrow cultures from NOX4 ${ }^{-1-}$ mice formed fewer osteoblastic colonies compared with WT cultures $(P<$ 0.05), suggesting a role for NOX4 in the maintenance of mesenchymal progenitor cell populations. These data suggest that NOX4 deletion is partially protective against $\mathrm{EtOH}$ effects on osteoblast differentiation, but may predispose bone to osteogenic impairments.

\section{Introduction}

Adult bone health is maintained by coordinated activities of bone resorption and bone formation, a process termed remodeling. Following osteoclastic bone resorption, preosteoblasts from the mesenchymal lineage infiltrate the resorbed surface and mature into osteoblasts (Martin and Seeman, 2008). Osteoblast maturation is characterized by the Runx2dependent expression of alkaline phosphatase (AP) along with expression of factors including osteocalcin and collagen 1a1 (Komori et al., 1997; Long, 2011). Remodeling osteoblasts predominately lie along the trabecular network of long bone (e.g., tibia and femur) metaphyses, but also generate bone on

This work was supported in part by the National Institutes of Health National Institute on Alcohol Abuse and Alcoholism [Grant R37 AA018282] (to M.J.J.R.), National Institutes of Health National Institute of General Medical Sciences [Grant T32 GM106999-01], the Systems Pharmacology and Toxicology Training Program, University of Arkansas for Medical Sciences (UAMS), and UAMS Children's University Medical Group (CUMG) Funds.

${ }^{1}$ Current affiliation: PinPoint Testing, Little Rock, Arkansas.

https://doi.org/10.1124/jpet.117.247262.

S This article has supplemental material available at jpet.aspetjournals.org. the periosteal (outer) surface to increase bone width (Orwoll, 2003). During mineralization, these cells become entrapped within the mineralized compartment and further mature into osteocytes that orchestrate bone turnover (Dallas and Bonewald, 2010). Disruptions in osteoblast maturation decrease bone mass (Komori et al., 1997; Rzonca et al., 2004), resulting in pathologies such as osteopenia or osteoporosis (Poole and Compston, 2006).

The canonical Wnt pathway promotes osteoblast differentiation and osteogenesis (Duan and Bonewald, 2016). Suppression of glycogen synthase kinase 3-beta by the Frizzled receptor/LRP$5 / 6$ receptor complex prevents degradation of beta-catenin, allowing beta-catenin to induce TCF-dependent transcriptional activity, specifically Runx2 activation (Long, 2011; Duan and Bonewald, 2016). Disruption of this pathway can perturb the balance of resorption and formation to decouple bone remodeling and thereby damage bone (Shankar et al., 2006, 2008; Chen et al., 2009; Alund et al., 2017).

Increases in oxidative stress are associated with decreased rates of bone formation and Wnt antagonism (Almeida et al., 2007a,b). Osteoblasts express NADPH oxidase-2 (NOX2) and

ABBREVIATIONS: ANOVA, analysis of variance; AP, alkaline phosphatase; BM, bone marrow; BS, bone surface; BV, bone volume; CFU-F, fibroblast colony forming unit; EtOH, ethanol; micro-CT, micro-computed tomography; MSC, mesenchymal stromal cell; NOX, NADPH oxidase; NOX2, NADPH oxidase-2; NOX4, NADPH oxidase-4; Ob, osteoblast; PF, pair fed; ROS, reactive oxygen species; RT-qPCR, reverse transcription quantitative polymerase chain reaction; TV, total volume; WT, wild type. 
NADPH oxidase-4 (NOX4), which generate reactive oxygen species (ROS) exclusively (Bedard and Krause, 2007; Schröder, 2015). NOX2 is a plasma membrane-bound enzyme that generates superoxide radical, which can be converted to hydrogen peroxide by superoxide dismutase (Bedard and Krause, 2007). The constitutively active NOX4 generates hydrogen peroxide, with NOX4 activity highly correlated with expression (Brandes et al., 2014). Additional data suggest that these enzymes mediate ROS-dependent activation of different intracellular signaling pathways involving members of the mitogen-activated protein (MAP) kinase cascade (Chen et al., 2008). Osteoblast precursors and mature osteoblasts express high levels of NOX4 mRNA transcripts, suggesting an influential role for NOX4 in regulation of osteoblastogenesis and osteoblast function (Ambe et al., 2014; Mercer et al., 2014).

Alcohol [ethanol (EtOH)] adversely targets bone (Gaddini et al., 2016). Reductions in bone formation by $\mathrm{EtOH}$ have been reported in both human and experimental studies (Nyquist et al., 1996; Chen et al., 2010; Mercer et al., 2014). Heavy alcohol use is directly associated with lower bone mineral density and increased fracture risk (Santori et al., 2008; González-Reimers et al., 2011). In animal studies, coadministration of the antioxidant glutathione precursor $\mathrm{N}$-acetylcysteine reverses EtOH's disruption of bone turnover, including the suppression of the Wnt pathway and osteogenic lineage commitment, implicating oxidative stress in EtOH's mechanism of action (Chen et al., 2010, 2011). In addition to suppressing bone formation, EtOH induces RANKL expression by producing excess ROS, thereby stimulating osteoclast differentiation and promoting resorption (Bai et al., 2005; Chen et al., 2006). Although bone loss can occur from an imbalance favoring either aspect of turnover, studies have found $\mathrm{EtOH}$ to suppress osteogenesis while promoting resorption (e.g., Callaci et al., 2009; Mercer et al., 2014; Yang et al., 2014). The direction of the uncoupling turnover can be model specific and dependent on physiologic status. For example, males and cycling females appear to be more susceptible to osteoclastogenic effects of EtOH due to lack of estrogen, whereas pregnant and postlactating female rats displayed only decreased bone formation markers (Shankar et al., 2006, 2008). The pro-osteoclastogenic mechanism of EtOH is dependent on NOX2 activity: loss of functional NOX2, via knockout of the essential component $\mathrm{p} 47^{\mathrm{phox}}$, prevented RANKL induction and osteoclastogenesis (Mercer et al., 2014). However, even in the absence of functional NOX2, EtOH suppressed osteoblast-mediated bone formation in mice (Mercer et al., 2014), yet the pan-NADPH oxidase (NOX) inhibitor diphenyleneiodonium blocked EtOH inhibition of bone formation in the rat (Chen et al., 2011). Given the finding that EtOH's suppression of bone formation is NOX2 independent, and NOX4's complimentary role in ROS generation in bone, we hypothesized that NOX4 is instrumental in suppressing osteoblast differentiation and/or function in response to $\mathrm{EtOH}$. The studies described herein employed global NOX4 deletion in male mice to determine NOX4's contribution to the reduction in bone quality induced by EtOH. In particular, EtOH-mediated changes in long bone microarchitecture, bone formation, and osteoblast maturation via antagonism of the Wnt pathway.

\section{Materials and Methods}

Animals. All experimental procedures involving the use of animals were approved by the Institutional Animal Care and Use Committee at the University of Arkansas for Medical Sciences and the Institutional Animal Care and Use Committee at Louisiana State University Health Sciences Center, New Orleans. Mice were housed in animal facilities approved by the Association for Assessment and Accreditation of Laboratory Animal Care. Thirteen-week-old male C57Bl/6J mice (Jackson Laboratories, Bar Harbor, ME) and NOX44 ${ }^{-1-}$ mice (B6.129-NOX $4^{t m 1 K k r} / \mathrm{J}$; Jackson Laboratories) were randomized to two groups and received either a $5 \% \mathrm{v} / \mathrm{v} \mathrm{EtOH}$ liquid diet (equaling $30 \%$ of total caloric intake) or a calorically matched pair-fed (PF) control using the Leiber-DeCarli liquid diet (35\% of energy from fat, $18 \%$ of energy from protein, and $47 \%$ of energy from carbohydrates; \#710260, Dyets, Inc., Bethlehem, PA). Additional NOX $4^{-1-}$ mice were given a standard rodent chow diet (Supplemental Figs. 2 and 3). $\mathrm{EtOH}$ was added to the Leiber-DeCarli diet by substituting carbohydrate calories until 30\% of total calories from EtOH was reached (Alund et al., 2016). This concentration was maintained for the 84 days of the experiment until sacrifice. Calorically matched $\mathrm{PF}$ feeding volumes were based on the previous day's consumption by the EtOH group. No significant rate of mortality was noted during the treatment phase. The final $N$ value for each group was as follows: wild-type (WT) PF, $N$ =4; WT EtOH, $N=5$; $\mathrm{NOX}^{-l-} \mathrm{PF}, N=5$; NOX $4^{-l-}$ EtOH, $N=6$; and $\mathrm{NOX}^{-1-}$ chow, $N=4$. At sacrifice, right and left femurs as well as vertebrae were harvested and frozen for total RNA extraction. The right tibias were fixed in $10 \%$ formalin for micro-computed tomography (micro-CT) analysis; left tibias were fixed in EtOH for histologic staining and dynamic histomorphometry analysis and histologic staining as previously described (Rzonca et al., 2004). Bone marrow (BM) mesenchymal stromal cells (MSCs) extracted from male C57Bl6/J, $\mathrm{NOX} 4^{-1-}$ mice, and p47 $7^{\text {phox }-1-}$ mice (B6N.129S2-Ncf $1^{\text {tm } 1 \text { Shl }} / \mathrm{J}$; Jackson Laboratories) aged 9 to 10 weeks were used for ex vivo osteoblast colony formation assays as described subsequently.

Micro-Computed Tomography. Formalin-fixed tibiae were imaged using high-resolution micro-CT according to current guidelines for the assessment of bone microarchitecture (Bouxsein et al., 2010). Right tibias were scanned using a Scanco MicroCT 40 (Scanco Medical AG, Brüttisellen, Switzerland) at $12 \mu \mathrm{m}$ voxel resolution. The trabecular compartment included a $2.8 \mathrm{~mm}$ region extending distally $1.24 \mathrm{~mm}$ from the primary spongiosa. All data were acquired at $70 \mathrm{kVp}, 114 \mathrm{~mA}$, and 200 millisecond integration time. The cancellous bone region was obtained in a blinded manner using a semiautomated contouring program that separated cancellous from cortical bone. The bone volume (BV)/total volume (TV) fraction percentage, trabecular thickness (in millimeters), trabecular separation (in millimeters), and trabecular number $(1 / \mathrm{mm})$ were calculated using previously published methods (Suva et al., 2008). For cortical bone of the tibia, the cortical compartment was a 1-mm-long region centered at the tibial midshaft. Total cross-sectional area $\left(\mathrm{mm}^{2}\right)$, medullary area $\left(\mathrm{mm}^{2}\right)$, and cortical thickness (in millimeters) were assessed. Bone was segmented from soft tissue using the same threshold for all groups: $247 \mathrm{mg} \mathrm{HA} / \mathrm{cm}^{3}$ for trabecular and $245 \mathrm{mg} \mathrm{HA} / \mathrm{cm}^{3}$ for cortical bone.

Histology and Bone Histomorphometry. Static and dynamic histomorphometry analyses were performed on methyl methacrylateembedded tibiae as previously described (Shankar et al., 2006). Then, $20 \mathrm{mg} / \mathrm{kg}$ calcein was injected 7 and 3 days prior to sacrifice $(n=4$ to 5 animals per group). Parameters in the tibial diaphysis and metaphysis were collected in a blinded fashion using a BH-2 digitizing morphometry system (Olympus, Center Valley, PA) and data were obtained by manual tracing (Osteometrics, Inc., Decatur, GA). Measurements of bone formation and resorption, including bone formation rate/bone surface (BS), mineralizing surface/BS, bone resorption rate/ $\mathrm{BS}$, and erosion surface/BS) were made analogously to standard dynamic bone histomorphometry in unstained sections under UV illumination (Dempster et al., 2013).

Similarly, two serial cross sections of each tibia were obtained, the first of which was stained with von Kossa and tetrachrome, the second of which was stained with AP (Shankar et al., 2006). All histomorphometric examinations were performed in a blinded, nonbiased manner using a computerized semiautomated OsteoMeasure system (Osteometrics, Inc., Atlanta, GA) as previously described (Shankar et al., 
2006). All measurements were confined to the secondary spongiosa and restricted to an area between 700 and $1500 \mu \mathrm{m}$ distal to the growth plate metaphyseal junction of the proximal tibia. A minimum of 25 fields in the proximal tibia was evaluated. Toluidine blue and Hoechst and eosin-stained sections were assessed to determine total tissue area, total bone area, total BS, and eroded surface. Tartrateresistant acid phosphatase staining was used to determine osteoclast number and osteoclast surface. Within the same region of interest, AP staining identified osteoblast surface, osteoblast number, and osteoblasts per bone area.

Western Blotting. Cellular proteins were extracted from frozen bone samples as described previously (Chen et al., 2008). NOX4 expression was detected using an antibody reactive to mouse NOX4 (Santa Cruz Biotechnology, Inc., Santa Cruz, CA), followed by incubation with goat anti-rabbit secondary antibody conjugated with horseradish peroxidase (Santa Cruz Biotechnology). Total protein per sample was detected by Amido black staining (Boston Bioproducts, Ashland, MA).

RNA Extraction and Reverse Transcription Quantitative Polymerase Chain Reaction (RT-qPCR). Marrow-depleted femurs were ground and mRNA extracted in TRIzol reagent and Qiagen RNAplus Easy Kit (Qiagen, Hilden, Germany). cDNA was made using BioRad iScript reverse transcriptase (BioRad, Hercules, CA). Gene expression was analyzed using SYBR-Green on ABI 7500 Real-Time PCR (American Bioanalytical, Natick, MA). Frozen lumbar vertebrae were crushed with a mortar and pestle and mRNA extracted in TRIzol reagent, followed by phase separation in 1-bromo-3-chloropropane and isopropanol/EtOH washes. Gene expression was analyzed using the Power SYBR-Green RNA-to-Ct 1-Step Kit (Applied Biosystems, Foster City, CA) on a Roche LightCycler 480 thermal cycler (ThermoFisher Scientific, Waltham, MA). Results were calculated normalized to 18 s ribosomal RNA.

Wnt Array. Three RNA samples from each treatment group were randomly selected and applied to a primer array (PAMM-043ZF-24; Qiagen) comprising 84 targets related to the canonical and noncanonical Wnt pathway. Results were normalized to an additional four housekeeping primers (Actb, B2m, Gapdh, Gusb, and Hsp90ab1). Delta $\mathrm{Ct}$ values $\left(\mathrm{Ct}_{\text {target }}-\mathrm{Ct}_{\text {housekeeping avg. }}\right.$ ) were analyzed for significant differences among groups by one-way analysis of variance (ANOVA), and expression levels were evaluated as fold change versus housekeeping $\left(2^{(- \text {deltaCt) }}\right)$ and fold change versus WT PF $\left(2^{(- \text {delta*deltaCt) }}\right)$. The absence of genomic DNA contamination was confirmed by a genomic DNA control amplification threshold value greater than 35 cycles, according to manufacturer's recommendations (Qiagen).

Cell Culture. Bone marrow was flushed from tibiae and femurs of 9- to 10 -week-old $\mathrm{NOX}^{-/-}, \mathrm{p} 47^{\text {phox-/- }}$, and WT male mice by centrifugation passed through a $70 \mu \mathrm{m}$ filter as previously described (Chen et al., 2010). BM cells were plated at 320,000 cells $/ \mathrm{cm}^{2}$ in parafilm-sealed flasks (Corning, Corning, NY) in alpha minimal essential medium (Gibco Thermo Fisher, Grand Island, NY) supplemented with $10 \%$ fetal bovine serum and $1 \%$ penicillin/streptomycin (Gibco Thermo Fisher) as biologically independent replicates within six independent experiments. The medium was allowed to equilibrate to incubator conditions $\left(37^{\circ} \mathrm{C}, 5 \% \mathrm{CO}_{2}\right)$ for at least 4 hours prior to plating and media were changed every 2 days (50\% volume) (Chen et al., 2010). L-ascorbate phosphate was added to the media following equilibration at a concentration of $50 \mu \mathrm{M}$ in the flask. Where indicated, EtOH was added to the media at a final concentration of $50 \mathrm{mM}$ in the flask. On day 14, cultures were fixed and stained for AP using an AP detection kit according to the manufacturer's protocol (procedure \#85; Sigma, St. Louis, MO) with Fast Blue or Fast Violet RR Salt (Sigma). AP colonies and fibroblast colony forming units (CFU-F) were enumerated. Cultures were then counterstained with Gill's hematoxylin and total colonies were enumerated (total CFU-F).

Statistical Analysis. Except where noted, differences between genotypes and $\mathrm{EtOH}$ treatment, along with any interaction effects, were determined using two-way ANOVA followed by a Student-NewmanKeuls post-hoc test. All analyses were performed at alpha $=0.05$ using
SigmaPlot version 11.0 (Systat Software, San Jose, CA) or Microsoft Excel (2016) and significance $(P<0.05)$ is reported as such. Summary statistics listed in the text are mean \pm S.E.M.

\section{Results}

The objective of this study was to test the overall hypothesis that NOX4 is instrumental in the in vivo suppression of bone formation by $\mathrm{EtOH}$, where mice lacking NOX4 activity would retain osteoblast differentiation and activity, tissue mineralization, and bone structure regardless of EtOH consumption. Male, C57Bl/6J mice homozygous for ablation of exon 4 of the NOX4 gene (B6.129-NOX4 $4^{\text {Tm1Kkr }} / \mathrm{J}$; Jackson Laboratories) (Carnesecchi et al., 2011) were investigated in comparison with WT mice of the same age and sex (commencing at the postpubertal age of 13 weeks when longitudinal growth had ceased). Following a 12-week exposure to either an EtOH liquid diet or calorically paired high-fat diet, tibias were collected and analyzed for structural phenotype of the trabecular (metaphysis) compartment and cortical midshaft (diaphysis) by micro-CT. Three-dimensional reconstructions of the trabecular bone region of interest are shown in Fig. 1.

As expected, EtOH consumption produced a significant decrease in fractional BV/TV in WT mice $(21.6 \% \pm 0.11 \%$ in WT PF vs. $16.1 \% \pm 0.45 \%$ in WT EtOH). However, and contrary to our hypothesis, NOX4 deletion did not protect bone in the trabecular (cancellous bone) compartment of mice consuming $\mathrm{EtOH}\left(18.7 \% \pm 1.1 \%\right.$ in $\mathrm{NOX} 4^{-/-} \mathrm{PF}$ vs. $10.9 \% \pm$ $1.8 \%$ in $\mathrm{NOX}^{-1-}$ EtOH) (Fig. 1A). Rather, NOX4 deletion exacerbated the EtOH effect (Fig. $1 \mathrm{~B}, P=0.014$ vs. WT EtOH). Trabecular number decreased significantly only in EtOH-fed $\mathrm{NOX}^{-1-}$ mice $(5.34 \pm 0.06$ and $4.99 \pm 0.16$ per $\mathrm{mm}$ for WT PF and $\mathrm{EtOH}$, respectively; $5.21 \pm 0.11$ and $3.06 \pm 0.49$ per $\mathrm{mm}$ for $\mathrm{NOX}^{-1-} \mathrm{PF}$ and $\mathrm{NOX} 4^{-1-}$ EtOH, respectively) with a corresponding borderline statistically significant increase $\left(P=0.08\right.$ compared with $\left.\mathrm{NOX} 4^{-1-} \mathrm{PF}\right)$ in trabecular spacing $(0.177 \pm 0.0042$ and $0.189 \pm 0.0083 \mathrm{~mm}$ for WT PF and WT EtOH, respectively; $0.178 \pm 0.0039$ and $0.363 \pm 0.068 \mathrm{~mm}$ for $\mathrm{NOX}^{-1-} \mathrm{PF}$ and $\mathrm{NOX} 4^{-1-} \mathrm{EtOH}$, respectively). Together, these results suggest that NOX4 deletion increases the susceptibility of the trabecular compartment to EtOH's adverse effects on bone.

In contrast, the cortical compartment at the tibia midshaft of $\mathrm{NOX}^{-1-}$ mice displayed a modest protection against the effect of EtOH treatment to decrease cortical bone parameters (Fig. 1, C and D). Despite a subtle but statistically significant decrease in cortical thickness in EtOH-fed WT mice $(P=0.04$ vs. WT PF), there was no significant decrease in cortical thickness in EtOH-fed NOX $4^{-l-}\left(P=0.167\right.$ vs. NOX $4^{-l-} \mathrm{PF}$; $0.204 \pm 0.0041$ and $0.190 \pm 0.0025 \mathrm{~mm}$ for WT PF and WT EtOH, respectively; $0.202 \pm 0.0064$ and $0.193 \pm 0.0044 \mathrm{~mm}$ for $\mathrm{NOX}^{-1-} \mathrm{PF}$ and NOX4 ${ }^{-l-}$ EtOH, respectively) (Fig. 1D, Ct. Th). However, EtOH-fed NOX $4^{-\prime-}$ mice had smaller bones overall, as indicated by a significant decrease in bone area $\left(0.336 \pm 0.0049\right.$ and $0.328 \pm 0.0088 \mathrm{~mm}^{2}$ for WT PF and WT EtOH, respectively; $0.326 \pm 0.0058$ and $0.294 \pm 0.014 \mathrm{~mm}^{2}$ for $\mathrm{NOX}^{-1-} \mathrm{PF}$ and NOX $4^{-1-}$ EtOH, respectively; $P=0.043$ $\mathrm{NOX}^{-1-} \mathrm{PF}$ vs. NOX4 $\left.{ }^{-1-} \mathrm{EtOH}\right)$, with genotype-driven decreases in total area $(0.596 \pm 0.017$ and $0.607 \pm$ $0.023 \mathrm{~mm}^{2}$ for WT PF and WT EtOH, respectively; $0.562 \pm$ 0.025 and $0.517 \pm 0.037 \mathrm{~mm}^{2}$ for $\mathrm{NOX}^{-1-} \mathrm{PF}$ and $\mathrm{NOX} 4^{-1-}$ EtOH, respectively; $P=0.27 \mathrm{NOX} 4^{-/-} \mathrm{PF}$ vs. NOX $4^{-/-} \mathrm{EtOH}$ ) 
A

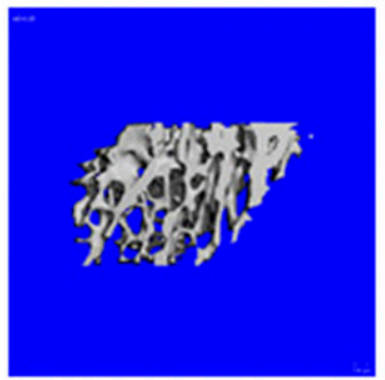

WT Pair Fed

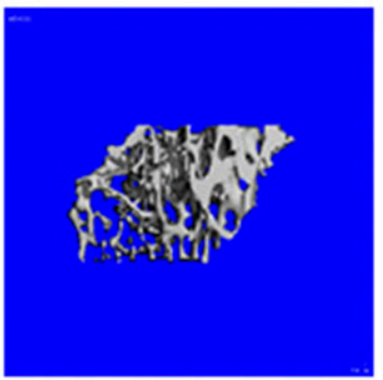

WT EtOH

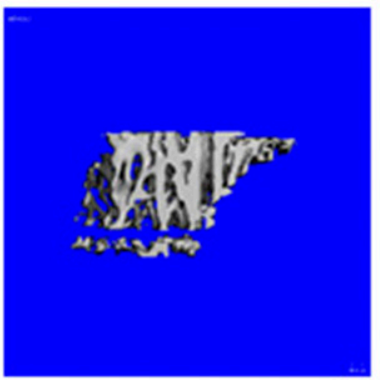

Nox4 Pair Fed

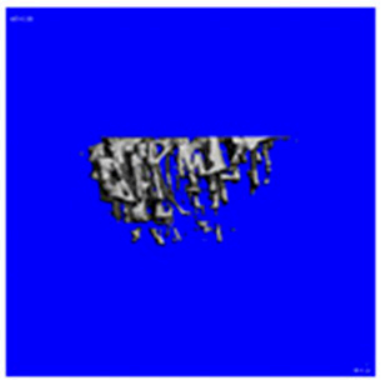

Nox4 EtOH

B BV/TV Tb.N Tb.Sp
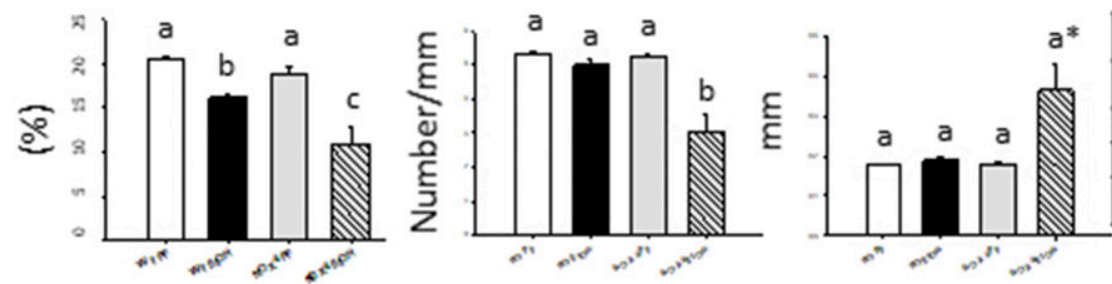

\begin{tabular}{|r|r|r|r|}
\hline \multicolumn{4}{|c|}{ 2-way ANOVA p-values: Trabecular analysis } \\
\hline & Genotype & $\underline{\text { Ethanol }}$ & Interaction \\
\hline BV/TV & 0.028 & 0.001 & 0.271 \\
\hline Tb.N. & 0.016 & 0.005 & 0.030 \\
\hline Tb.Sp. & 0.095 & 0.066 & 0.101 \\
\hline
\end{tabular}

C

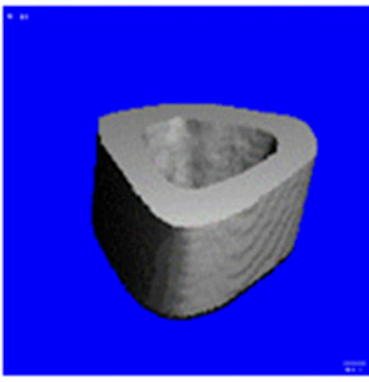

WT Pair Fed

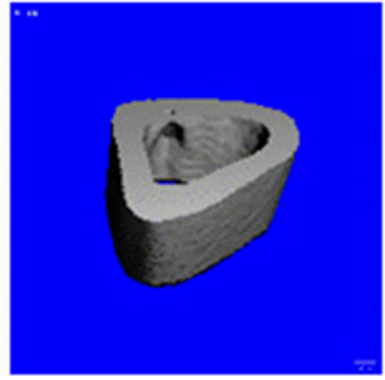

WT EtOH

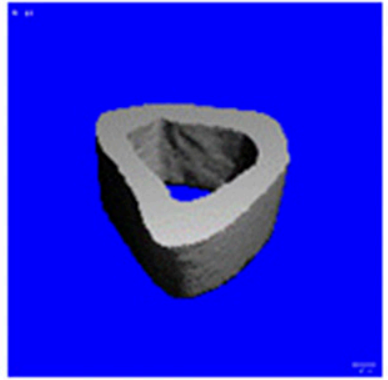

Nox4 Pair Fed

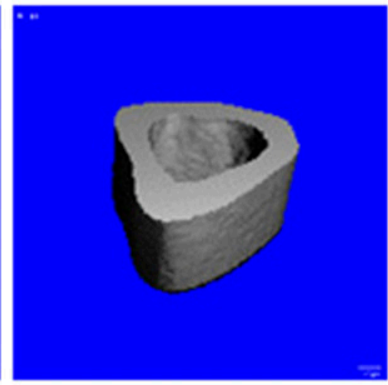

Nox4 EtOH

D

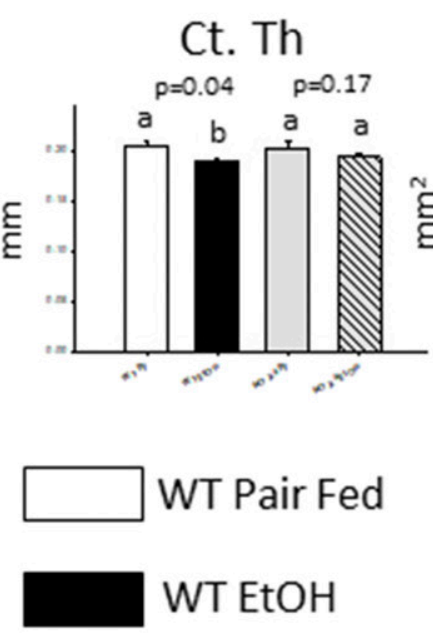

B. $\mathrm{Ar}$
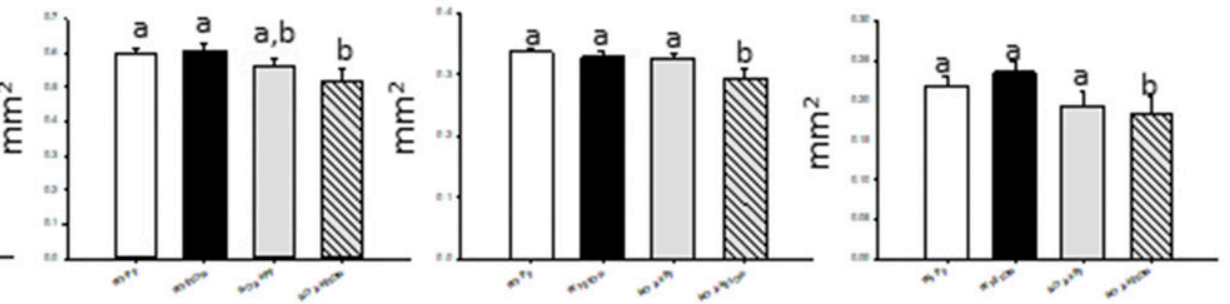

\begin{tabular}{|r|r|r|r|}
\hline \multicolumn{4}{|c|}{ 2-way ANOVA p-values: Cortical analysis } \\
\hline & Genotype & $\underline{\text { Ethanol }}$ & Interaction \\
\hline Ct.Th. & 0.889 & $\mathbf{0 . 0 2}$ & 0.269 \\
\hline T.Ar. & $\mathbf{0 . 0 4 1}$ & 0.557 & 0.326 \\
\hline B.Ar. & $\mathbf{0 . 0 4 7}$ & 0.066 & 0.269 \\
\hline Med.Ar & $\mathbf{0 . 0 4 4}$ & 0.832 & 0.437 \\
\hline
\end{tabular}

Fig. 1. $\mathrm{NOX}^{-1-}$ mice have reduced trabecular BV but sustained cortical bone mass with EtOH. (A) Micro-CT images of the trabecular compartment (12 $\mu \mathrm{m}$ resolution). (B) Micro-CT-evaluated trabecular bone parameters: fractional BV/TV; trabecular number (Tb.N); trabecular spacing (Tb.Sp). For each group: WT PF, $N=3$; WT EtOH, $N=4$; $\mathrm{NOX}^{-1-} \mathrm{PF}, N=3$; $\mathrm{NOX} 4^{-1-} \mathrm{EtOH}, N=5$. (C) Micro-CT images of cortical compartment (12 $\mu \mathrm{m}$ resolution). (D) Micro-CT-evaluated cortical bone parameters: cortical thickness (Ct.Th); total area (T.Ar); bone area (B.Ar); medullary area (M.Ar). For each group: WT PF, $N=4$; WT EtOH, $N=5$; NOX $4^{-1-} \mathrm{PF}, N=4$; $\mathrm{NOX}^{-1-} \mathrm{EtOH}, N=5$. Statistical significance was determined with two-way ANOVA; values with different letter superscripts are statistically different from each other $(P<0.05$, Student-Newman-Keuls post hoc test; $* P=0.08$ for comparison of mean to $\left.\mathrm{NOX} 4^{-1-} \mathrm{PF}\right)$ 
TABLE 1

Dynamic histomorphometry parameters

Mean \pm S.E.M. Values with superscript letters a and b are statistically different from each other $(P<0.05$, two-way ANOVA, Student-Newman-Keuls post hoc test); superscript $\dagger$ denotes significant main effect of genotype (WT vs. NOX4 ${ }^{-l-}$ ); superscript $\ddagger$ denotes significant main effect of $\mathrm{EtOH}$ (PF vs. EtOH) $(P<0.05$, two-way ANOVA).

\begin{tabular}{lcccc}
\hline \multicolumn{1}{c}{ Parameter } & WT PF $(n=4)$ & WT EtOH $(n=5)$ & NOX $^{-/-}$PF $(n=5)$ & NOX $^{-1-}$ EtOH $^{\prime}(n=5)$ \\
\hline T.Ar $\left(\mathrm{mm}^{2}\right)$ & $2.23 \pm 0.15$ & $2.38 \pm 0.12$ & $2.24 \pm 0.25$ & $2.21 \pm 0.20$ \\
B.Ar $\left(\mathrm{mm}^{2}\right)$ & $0.51 \pm 0.004^{\dagger, \mathrm{a}}$ & $0.60 \pm 0.08^{\dagger, \mathrm{a}}$ & $0.41 \pm 0.02^{\mathrm{b}}$ & $0.42 \pm 0.04^{\mathrm{b}}$ \\
BV/TV $(\%)$ & $23.23 \pm 1.46^{\dagger, \dagger, \mathrm{a}}$ & $20.49 \pm 1.31^{\dagger, \ddagger, \mathrm{a}, \mathrm{b}}$ & $20.89 \pm 1.36^{\mathrm{a}, \mathrm{b}}$ & $17.01 \pm 1.25^{\mathrm{b}}$ \\
$\mathrm{MAR}(\mu \mathrm{m} / \mathrm{d})$ & $2.78 \pm 0.25$ & $2.93 \pm 0.33$ & $3.16 \pm 0.31$ & $3.41 \pm 0.21$ \\
BFR/BS $\left(\mu \mathrm{m}^{3} / \mu \mathrm{m}^{2} / \mathrm{d}\right)$ & $0.72 \pm 0.21$ & $0.62 \pm 0.17$ & $0.78 \pm 0.12$ & $0.62 \pm 0.18$ \\
\hline
\end{tabular}

B.Ar, bone area; BFR/BS, bone surface-normalized bone formation rate; BV/TV, fractional bone volume; MAR, mineral apposition rate; T.Ar, total area.

and medullary area $\left(0.343 \pm 0.014\right.$ and $0.345 \pm 0.0060 \mathrm{~mm}^{2}$ for WT PF and WT EtOH, respectively; $0.313 \pm 0.015$ and $0.318 \pm 0.021 \mathrm{~mm}^{2}$ for $\mathrm{NOX}_{4}^{-/-} \mathrm{PF}$ and $\mathrm{NOX}^{-/-} \mathrm{EtOH}$, respectively; $P=0.69 \mathrm{NOX}^{-/-} \mathrm{PF}$ vs. NOX4 $\left.{ }^{-/-} \mathrm{EtOH}\right)$ compared with WT controls (Fig. 1D, T.Ar, B.Ar, and Med.Ar).

To determine whether the differences in $\mathrm{BV}$ were the result of diminished bone formation, mice were subcutaneously injected with calcein label 7 and 3 days prior to sacrifice. Dynamic histomorphometry parameters of the mineral apposition rate and bone surface area-normalized bone formation rate were not significantly altered by genotype or EtOH treatment (Table 1). However, significant decreases in bone area with NOX4 deletion $\left[0.556 \mathrm{~mm}^{2}\right.$ for WT (PF and EtOH average $)], 0.415 \mathrm{~mm}^{2}$ for $\mathrm{NOX}^{-1-}[(\mathrm{PF}$ and EtOH average), $P<0.05$ for genotype effect, two-way ANOVA)] and significantly lower TV-normalized bone WT volume (BV/TV) were observed in $\mathrm{NOX}^{-1-}$ EtOH-treated animals compared with WT PF animals (23.24\% for WT PF, $17.01 \%$ for $\mathrm{NOX}^{-/} \mathrm{EtOH}$, $P<0.05$ ). NOX4 deletion significantly decreased the total number of osteoblasts (Table 2) compared with WT (93.0 \pm 7.3 in WT, $58.2 \pm 6.9$ in NOX $4^{-/-} ; P<0.01$ for the main genotype effect, two-way ANOVA). Among the mice treated with EtOH, NOX $4^{-1-}$ mice had fewer osteoblasts compared with WT PF mice $\left(99.3 \pm 10.7\right.$ in WT PF, $48.2 \pm 8.9$ in NOX $4^{-1-}$ EtOH, $P<$ $0.05)$ and fewer osteoblasts compared with WT EtOH-fed mice with borderline significance $(86.6 \pm 12.3$ in WT PF, $P=0.06)$. When normalized to BS (i.e., bone surface area-normalized osteoblast surface), the genotype difference was no longer statistically significant. EtOH did not significantly decrease the osteoblast number overall. However, the total area-normalized osteoblast $(\mathrm{Ob})$ count was significantly decreased by NOX4 deletion (Table 2, $160.3 \pm 9.2 \mathrm{Ob} / \mathrm{mm}^{2}$ in WT, $104.5 \pm 11.8 \mathrm{Ob} / \mathrm{mm}^{2}$ in NOX $4^{-/-} ; P<0.01$ for the main genotype effect, two-way ANOVA), and $\mathrm{NOX}^{-/-}$animals treated with EtOH had fewer total area-normalized osteoblast counts compared with the other three groups (174.0 \pm $11.5 \mathrm{Ob} / \mathrm{mm}^{2} \mathrm{Ob} / \mathrm{mm}^{2}$ in WT PF, $146.6 \pm 12.0 \mathrm{Ob} / \mathrm{mm}^{2}$ in WT EtOH, $129.9 \pm 14.0$ in NOX4 $4^{--} \mathrm{PF}, 79.05 \pm 10.5 \mathrm{Ob} / \mathrm{mm}^{2}$ in $\mathrm{NOX}^{-l-} \mathrm{EtOH}, P<0.05$, two-way ANOVA). The data suggest an exacerbation of the suppressive effect of $\mathrm{EtOH}$ on osteoblast differentiation, and a decrease in total osteoblast activity, in the absence of NOX4. No significant differences were observed among treatments or genotypes for measures of osteoclast prevalence or activity (Supplemental Table 1).

To identify intracellular mechanisms contributing to the observed phenotypes, total RNA was extracted from femur cortices and vertebral bone, and gene expression (relative to 18s rRNA) was determined by RT-qPCR (Fig. 2). As was observed via micro-CT, EtOH appeared to have a compartment-specific effect. Vertebral bone, which is largely trabecular, but not the femur cortical midshaft, appeared to be sensitive to the antiosteogenic effect of EtOH regardless of NOX4 expression. In cortical bone, EtOH-fed WT mice displayed significantly reduced expression of the osteogenic genes osteocalcin (Bglap) (Fig. 2A) and collagen 1a1 (Col1a1) (Fig. 2B) compared with WT PF controls $(P=0.007$ and 0.002 , respectively), whereas EtOH had no effect on the expression of these genes in NOX4 ${ }^{-1-}$ mice $(P=0.612$ and 0.997 , for Bglap and Col1a1, respectively). EtOH did not have a NOX4dependent effect in vertebral bone: overall, $\mathrm{EtOH}$ suppressed AP expression with borderline statistical significance (Fig. 2C, $P=0.08$ for main effect of EtOH, two-way ANOVA) and suppressed collagen 1a1 expression (Fig. $2 \mathrm{D}, P=0.05$ for main effect of EtOH, two-way ANOVA).

NOX2 and RANKL expression are related to EtOH-induced bone resorption (Mercer et al., 2014). In cortical samples from WT mice, EtOH upregulated mRNA expression of NOX2 compared with $\mathrm{PF}$ mice (Fig. $2 \mathrm{E})(P<0.003)$. This was

TABLE 2

Static histomorphometry parameters of bone formation

Means \pm S.E.M. Values with superscript letters a and b are statistically different from each other $(P<0.05$, two-way ANOVA, Student-Newman-Keuls post hoc test); superscript + denotes significant main effect of genotype (WT vs. NOX4 ${ }^{-1-}$ ); superscript $¥$ denotes significant main effect of EtOH ( $\mathrm{PF}$ vs. EtOH) $(P<0.05$, two-way ANOVA).

\begin{tabular}{|c|c|c|c|c|}
\hline Parameter & WT PF $(n=4)$ & WT EtOH $(n=5)$ & $\mathrm{NOX}_{4}{ }^{-1-} \mathrm{PF}(n=5)$ & $\mathrm{NOX}_{4}{ }^{-1-} \mathrm{EtOH}(n=5)$ \\
\hline T.Ar $\left(\mathrm{mm}^{2}\right)$ & $0.569 \pm 0.044$ & $0.595 \pm 0.070$ & $0.525 \pm 0.0001$ & $0.595 \pm 0.043$ \\
\hline B. $\mathrm{Ar}\left(\mathrm{mm}^{2}\right)$ & 0.071 & 0.0040 & $0.050 \pm 0.0$ & $48 \pm 0.017$ \\
\hline $\mathrm{N} . \mathrm{Ob}$ (number) & $99.25 \pm 10.7^{\dagger, \mathrm{a}}$ & $86.60 \pm 12.3^{\dagger, \mathrm{a}}$ & $68.20 \pm 7.35^{\mathrm{a}, \mathrm{b}}$ & $0 \pm 8.87^{b}$ \\
\hline $\mathrm{BS} / \mathrm{BV}(\%)$ & $58.67 \pm 3.88$ & $59.07 \pm 1.83$ & $50.82 \pm 4.89$ & $58.51 \pm 7.30$ \\
\hline Ob.S/BS (\%) & $15.73 \pm 1.52$ & $16.57 \pm 1.95$ & $20.16 \pm 2.27$ & $13.76 \pm 1.46$ \\
\hline N.Ob/T.Ar $\left(1 / \mathrm{mm}^{2}\right)$ & $174.05 \pm 11.5^{\dagger, \ddagger, \mathrm{a}}$ & $146.57 \pm 12.0^{\dagger, \nRightarrow, \mathrm{a}}$ & $129.91 \pm 14.0^{\mathrm{a}}$ & $79.05 \pm 10.5^{\mathrm{b}}$ \\
\hline
\end{tabular}

B.Ar, bone area; BS/BV, volume-normalized bone surface area; N.Ob, osteoblast number; N.Ob/T.Ar, number of osteoblasts per total area; Ob.S/BS, bone surface area-normalized osteoblast surface; T.Ar, total area. 


\section{A \\ Cortical: Bglap

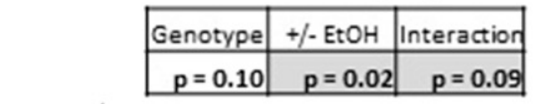

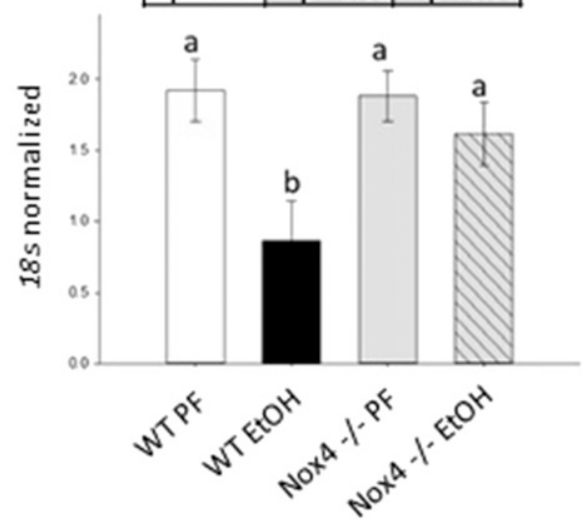

C Vertebral: Alpl

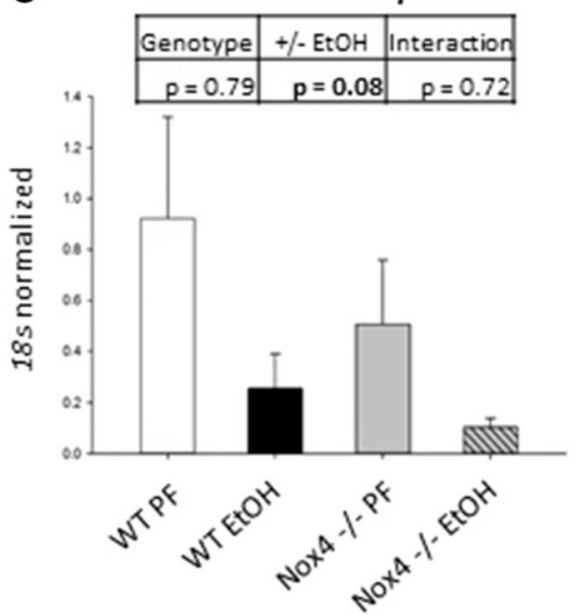

Cortical: Nox2
\begin{tabular}{|r|c|c|}
\hline Genotype & $+/$ - EtOH & Interaction \\
\hline$p=0.21$ & $p=0.02$ & $p=0.02$ \\
\hline
\end{tabular}

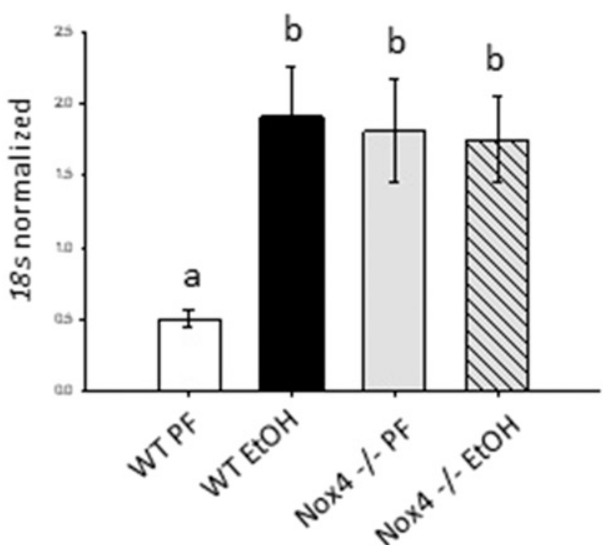

B
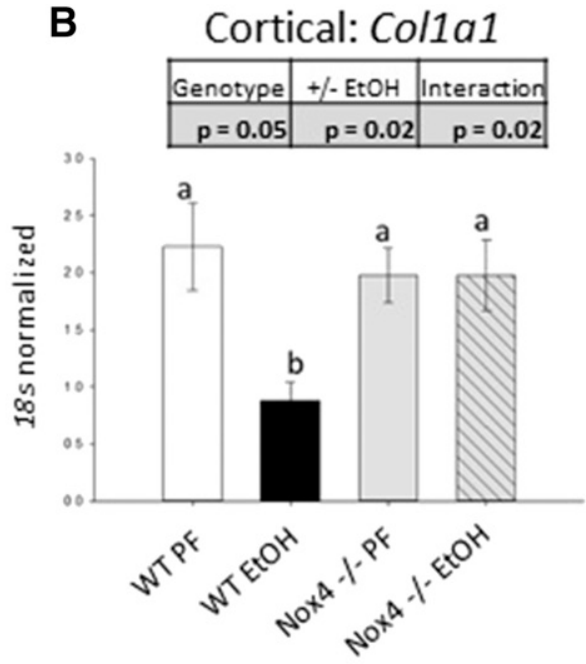

D Vertebral: Col1a1
\begin{tabular}{|r|r|l|}
\hline Genotype & ++ - EtOH & Interaction \\
\hline$p=0.20$ & $p=0.05$ & $p=0.79$ \\
\hline
\end{tabular}

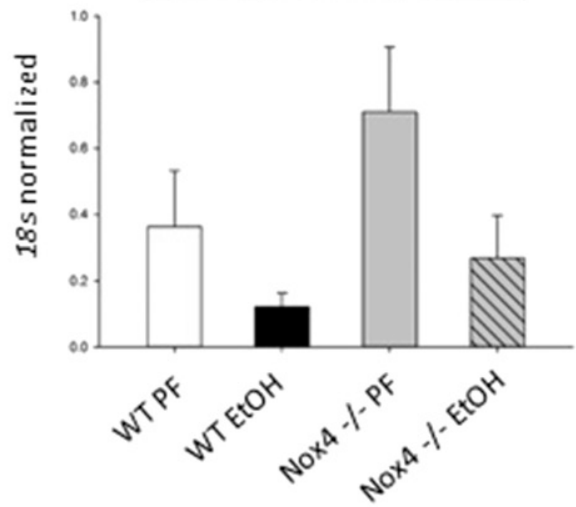

D Cortical: RANKL
\begin{tabular}{|l|l|l|}
\hline Genotype & + - EtOH & Interaction \\
\hline$p=0.012$ & $p<0.001$ & $p=0.12$ \\
\hline
\end{tabular}

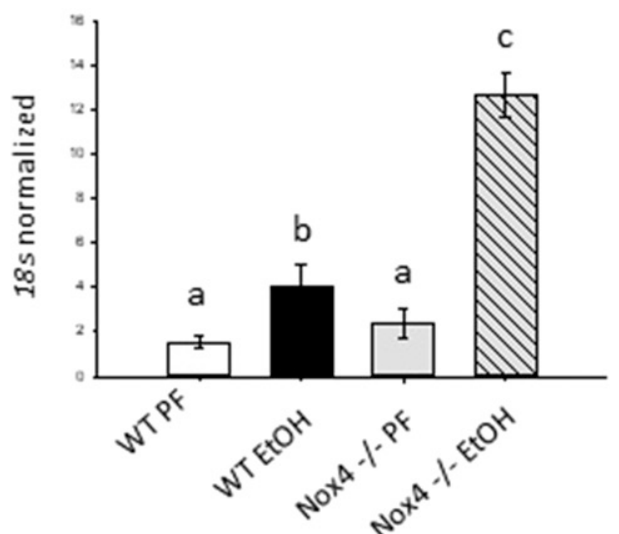

Fig. 2. NOX4 deletion is protective of EtOH-induced antiosteogenic effects in cortical but not trabecular bone. (A) Cortical bone-derived osteocalcin (Bglap); (B) cortical bone-derived collagen 1a1 (Col1a1); (C) vertebral bone-derived AP (Alpl); (D) vertebral bone-derived collagen 1a1 (Col1a1); (E) cortical bone-derived NOX2 (Nox2); and (F) cortical bone-derived RANKL (RANKL). For each group, $n=3-5$. Statistical significance was determined with two-way ANOVA; values with different letter superscripts are statistically different from each other $(P<0.05$, Student-Newman-Keuls post hoc test). 
A

NOX4

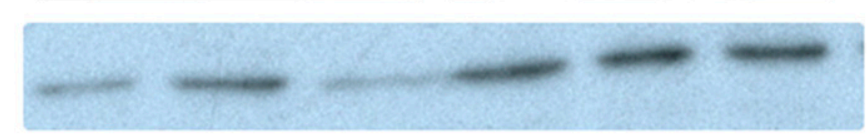

Fig. 3. EtOH increases expression of NOX4 protein in WT mice. Representative samples ( $n=3 \mathrm{PF}$ and three $\mathrm{EtOH})$ of total protein from WT mice isolated following aspiration of BM. (A) anti-NOX4 antibody (Santa Cruz Biotechnologies). Amido black staining of total protein. (B) densitometric quantification of western immunoblots. ${ }^{*} P<0.05$, Student's unpaired $t$ test.

\section{B}

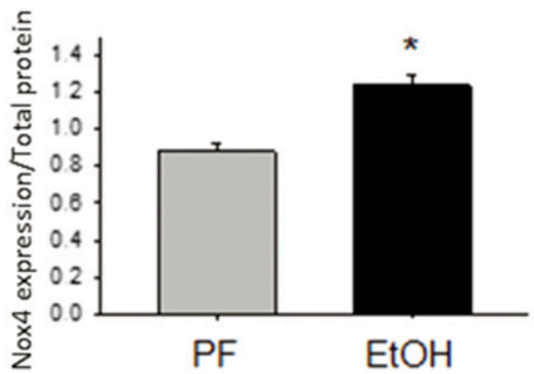

accompanied by significant increases in NOX2 expression in both PF and EtOH-fed NOX4 ${ }^{-1-}$ mice compared with WT PF, although there was no effect of $\mathrm{EtOH}$ on $\mathrm{NOX} 4^{-1-}$ mice. As expected, EtOH significantly upregulated the expression of $R A N K L$ in both WT and NOX4 $4^{-1-}$ mice $(P<0.0001$ for the main effect of EtOH, two-way ANOVA) (Fig. 2F). Notably, $\mathrm{NOX}^{-1-}$ animals showed higher expression of RANKL compared with WT ( $P=0.012$ for the main effect of genotype, two-way ANOVA), with $\mathrm{NOX}^{-1-} \mathrm{EtOH}$ mice showing a potentiated increase in $R A N K L$ expression $(P<0.001$ compared with WT EtOH). Consistent with previous data showing upregulation of NOX4 mRNA and protein in EtOH-treated rats (Chen et al., 2011), NOX4 protein expression increased in WT, EtOH-fed mice (Fig. 3).

A critical driver of osteoblast differentiation is the canonical Wnt pathway (Duan and Bonewald, 2016). To determine whether EtOH affects aspects of this pathway in vivo, vertebral mRNA ( $n=3$ for each group) was applied to a Qiagen Wnt pathway array (Qiagen) that assays 84 separate Wnt targets. Expression levels of each gene were normalized to expression levels of internal housekeeping genes (Actb, B2m, Gapdh, Gusb, and Hsp90ab1) (Fig. 4A), and target gene up- or downregulation was determined relative to WT PF samples (Fig. 4B). There was no significant difference among the proportions of up- and downregulated targets in the three groups relative to WT PF ( $\chi^{2}, P=0.446$ ). However, one-way ANOVA of the housekeeping-adjusted values for all four groups identified statistically significant expression variation for 11 gene targets: three Wnt ligand molecules (Wnt3, Wnt4, and Wnt6); two Frizzled receptor genes (Fzd5 and Fzd8); beta catenin (Ctnnb1); four genes related to beta-catenin accumulation (Cnsk2a1), degradation (Gsk3b and Fbxw4), and DNA binding (Tcfl7); and Wnt signaling target gene Ccnd2.

To confirm these results, trabecular mRNA expression was evaluated for individual targets using RT-qPCR and results were analyzed by two-way ANOVA (Fig. 5). As a main effect, $\mathrm{EtOH}$ was found to significantly suppress expression of the gene encoding Wnt target cyclin D2 (Ccnd2) and a suppressive effect approached statistical significance for Frizzled receptor8 ( $F z d 8$, the main effect of EtOH $P=0.10$, two-way ANOVA). Expression of beta catenin (Cttnb1) mRNA was nonsignificantly upregulated by EtOH in both groups $(P=0.11)$. Deletion of NOX4 produced a borderline significant main effect in two-way ANOVA seen in Ccnd2 expression (the main effect of genotype $P=0.07$, two-way ANOVA) and borderline significant suppression of Wnt6 (the main effect of genotype $P$ $=0.06)$. No significant effects were found for expression of Fbxw4 or Fzd8; Wnt3 and Wnt4 mRNA did not amplify sufficiently in RT-qPCR for the purpose of analysis. Thus, in contrast to the cortical data where the effects of EtOH and NOX4 deletion appear to interact, trabecular effects of $\mathrm{EtOH}$ and NOX4 appeared to be independent.

To further elucidate the effect of $\mathrm{EtOH}$ on osteoblast differentiation in the context of NOX4 deletion, osteoblasts 


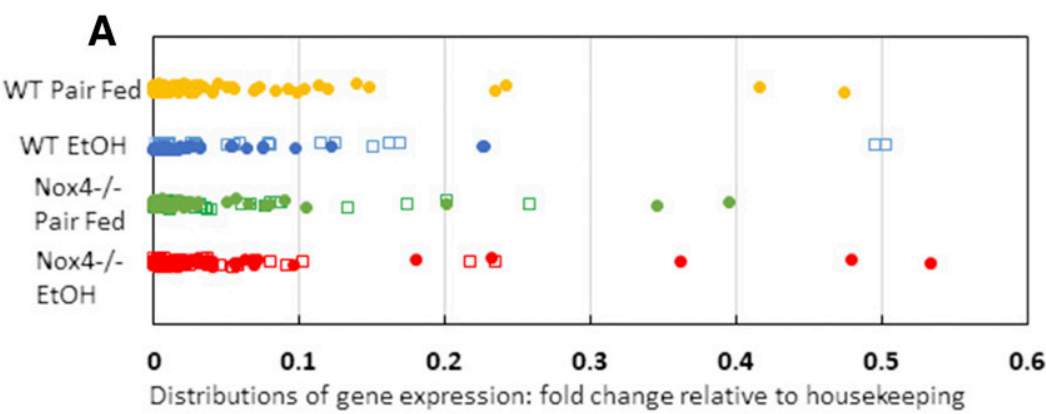

B
Fig. 4. Distribution of Wnt pathway array gene expression among treatment groups. (A) Fold change in gene expression relative to housekeeping genes (Actb, B2m, Gapdh, Gusb, and Hsp90ab1). (B) Relative changes in gene expression (upregulated or downregulated) in groups relative to WT PF. Open square: upregulated; closed circle: downregulated. Distributions of relative gene expression not statistically significant $\left(P>0.05 ; \chi^{2}\right) ; n=3$ from each group.

Number of genes up or downregulated relative to WT Pair Fed

\begin{tabular}{|c|l|l|l|}
\hline & $\square$ Upregulated & Downregulated & Total \\
\hline WT EtOH & 30 & 48 & 78 \\
\hline Nox4 Pair Fed & 34 & 49 & 83 \\
\hline Nox4 EtOH & 38 & 41 & 79 \\
\hline
\end{tabular}

were differentiated from BM-MSC primary cultures. BMMSCs from male and female, 9- to 10 -week-old mice were flushed from the femur and tibia BM and incubated in sealed flasks for 14 days in the presence of $50 \mu \mathrm{M}$ l-ascorbate phosphate alone or with $50 \mu \mathrm{M}$ l-ascorbate plus $50 \mathrm{mM}$ EtOH. At the end of the treatment period, cell cultures were assayed for CFU-Fs by Gill's hematoxylin and for AP as an indicator of osteoblast colony forming units (AP + CFU-Fs) (Fig. 6). BM-MSCs derived from NOX $4^{-1-}$ mice consistently developed fewer CFU-Fs compared with BM-MSCs derived from WT mice $(6.80 \pm 0.472$ colonies vs. $18.01 \pm 1.36$ colonies per 1 million cells plated, respectively; the main effect of genotype $P<0.0001$, two-way ANOVA). In contrast, EtOH had no significant effect on the number of CFU-Fs within each genotype (Fig. 6A). However, $50 \mathrm{mM} \mathrm{EtOH}$ treatment revealed a differential effect with respect to AP + CFU-Obs. Normalized to hematoxylin-positive colonies, AP + CFU-F counts were suppressed by EtOH in WT cultures $(75.6 \%$ in untreated, $52.8 \%$ in EtOH-treated cultures), whereas $\mathrm{EtOH}$ treatment was nonsignificantly increased in AP + CFU-Fs in $\mathrm{NOX}^{-1-}$ cultures $(66.9 \%$ in untreated, $76.1 \%$ in EtOHtreated cultures, $P$ value for interaction $=0.006$, two-way ANOVA) (Fig. 6, B and C). Colonies derived from BM harvested from $\mathrm{p} 47^{\text {phox-l- }}$ mice (B6N.129S2-Ncf1 $1^{\text {tm } 1 S h l} / \mathrm{J}$; Jackson Laboratories), of the same age and sex, lacking $\mathrm{p} 47^{\text {phox }}$ (an essential signaling component of NOX2) were not protected against EtOH's suppression of $\mathrm{AP}(P<0.001 \mathrm{vs}$. p47 $7^{\text {phox-l- }}$ without EtOH) (Fig. 6D). Together, these data suggest an overall support of osteoblast colony formation, but sensitivity to the EtOH-mediated suppression of osteoblast maturation, in WT BM-MSCs compared with those lacking NOX4.

\section{Discussion}

Chronic alcohol consumption can lead to a variety of health issues, including increased risk of osteopenia and bone fracture (Santori et al., 2008; González-Reimers et al., 2011). $\mathrm{EtOH}$ increases bone resorption and suppresses osteogenesis in part by increasing oxidative stress in osteoblasts (Chen et al., 2010). This mechanism appears to be dependent on the metabolism of EtOH by alcohol dehydrogenase (Mercer et al., 2014). EtOH metabolism by alcohol dehydrogenase increases the cellular pool of NADPH. In response, elevated activity of the NOX enzymes, particularly NOX4 and NOX2, produce excess superoxide and hydrogen peroxide via the action of superoxide dismutase (Schröder, 2015). Hydrogen peroxide can prevent murine osteoblast differentiation and expression of AP (Atashi et al., 2015). The finding that antioxidant administration can robustly prevent EtOH's suppression of bone homeostasis confirms the involvement of ROS in EtOH's mechanism of action (Shankar et al., 2008; Chen et al., 2010; Alund et al., 2017). However, the degrees to which different NOX enzymes contribute to EtOH's alterations of bone phenotype are largely unresolved.

NOX4 and NOX2 drive oxidative stress in bone cells in a complimentary manner, since pan-inhibition of NOX2 and NOX4 by diphenyleneiodonium reduces hydrogen peroxide generation in the mouse ST2 mesenchymal cell line (Mercer et al., 2014). We previously demonstrated that functional NOX2 deletion prevents EtOH-mediated increases in bone resorption, but does not prevent the suppression of bone formation: despite loss of functional NOX2, EtOH significantly downregulated serum osteocalcin, indicating a suppression of anabolic activity in bone (Mercer et al., 2014). These results implied a central role of the predominately expressed NOX4 in EtOH's impairment of bone homeostasis. Hence, this study was designed to determine the contribution of NOX4 activity to the suppression of bone formation with chronic EtOH consumption utilizing a global NOX4 knockout mouse model.

NOX4 deletion has previously been suggested to support bone mass. Specifically, NOX $4^{-1-}$ mice were reported to have a $\sim 30 \%$ increase in trabecular BV despite no significant change in histologic osteoblast parameters (Goettsch et al., 2013). Contrary to those observations, the micro-CT results presented herein (Fig. 1, A and B) indicate no change in trabecular bone mass with NOX4 deletion alone, but an exacerbated effect of EtOH to reduce trabecular bone mass in $\mathrm{NOX}^{-1-}$ mice. Differences between these studies may include the use of male mice and the age at start of treatment [13 weeks; the age and sex of intact mice used by Goettsch et al. (2013) is not noted] as well as the genetics of NOX4 deletion. Whereas Goettsch et al. (2013) deleted NOX4 via ablation of exons 1 and 2, the mice in the current study had exon 4 deleted (Carnesecchi et al., 2011). The latter method prevented amplification of mRNA targeting exon 4 (Supplemental Fig. 1) and was 
A

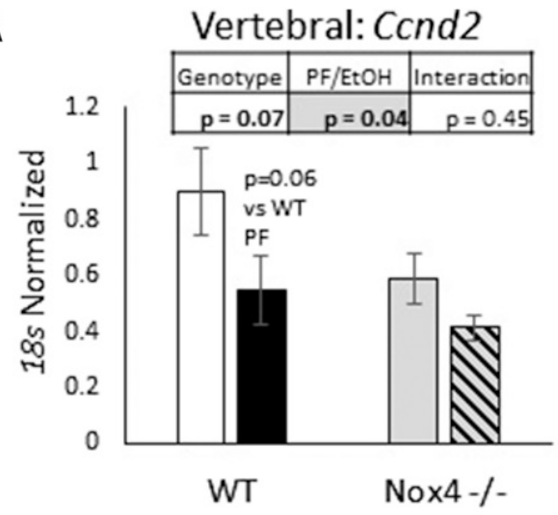

C

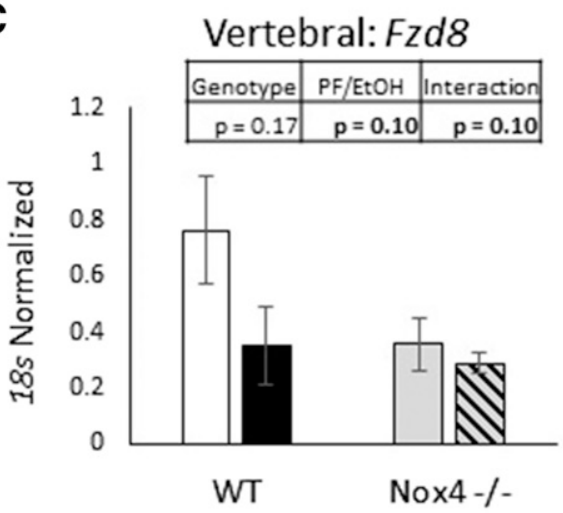

E

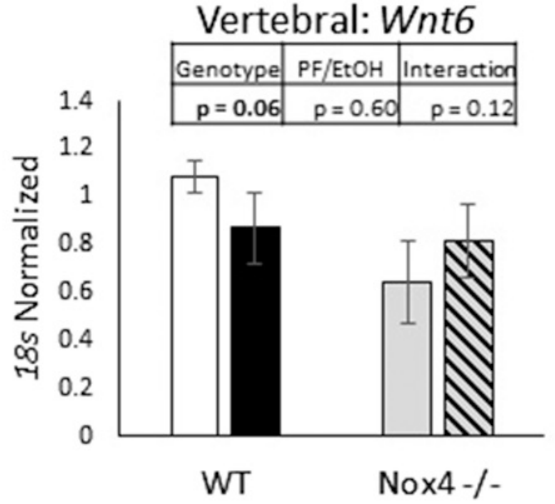

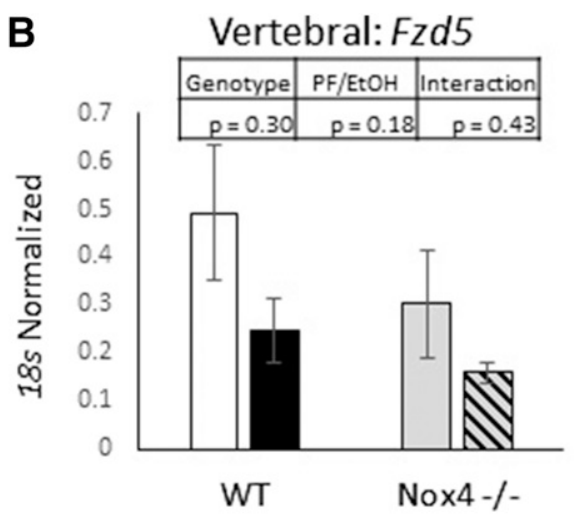

D

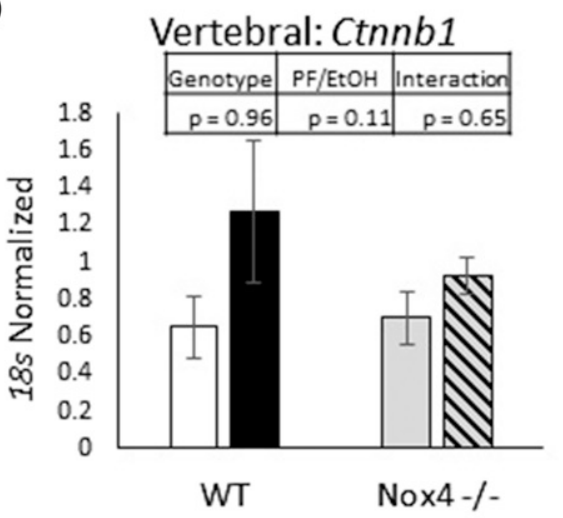

F

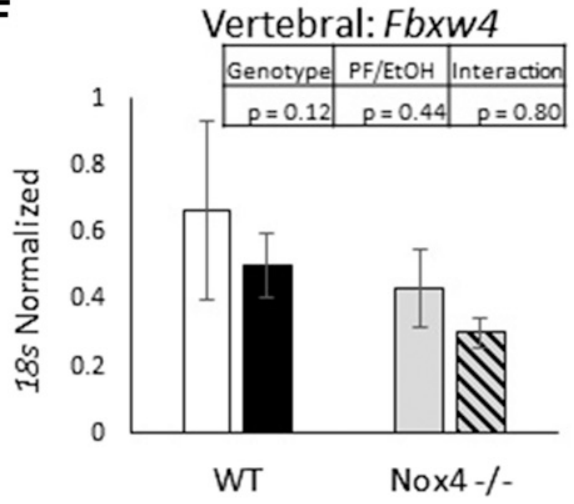

Nox $4 \%$ Pair Fed

Fig. 5. EtOH significantly downregulates targets of the Wnt pathway in vertebral bone. (A) Cyclin D2 (Ccnd2); (B) Frizzled-5 precursor (Fzd5); (C) Frizzled-8 precursor $(F z d 8)$; (D) Beta-catenin (Cttnb1); (E) Wnt-6 (Wnt6); and (F) F-box/WD repeat-containing protein 4 (Fbxw4). Results analyzed by two-way ANOVA, significant main effects are highlighted and in bold $(P<0.05)$; borderline significant effects $(P \leq 0.10$ in bold only). For each group: WT $\mathrm{PF}, n=4$; WT EtOH, $n=5$; NOX4 $4^{-1-} \mathrm{PF}, n=5$; NOX $4^{-1-} \mathrm{EtOH}, n=6$.

shown by others to abolish NOX4 expression in the spleen, kidney, and lung (Carnesecchi et al., 2011). Further investigation into alternate mechanisms associated with loss of NOX4, including compensatory NOX2 activity (suggested by increases in NOX2 mRNA) (Fig. 2E) or NOX1 (Wittrant et al., 2009) expression, are ongoing in our laboratory. Loss of NOX4 activity does not necessarily limit oxidative stress with $\mathrm{EtOH}$, which may also be associated with increases in mitochondrial-derived ROS. Addition of a NOX4-specific inhibitor, plumbagin, did not significantly prevent the accumulation of hydrogen peroxide in cultured, EtOH-treated ST2 cells (Mercer et al., 2014).
Micro-CT data indicated that cortical bone in $\mathrm{NOX}^{-1-}$ mice was less susceptible to bone loss following $\mathrm{EtOH}$ exposure. These mice had smaller tibias overall (i.e., lower total crosssectional area) but maintained cortical bone thickness compared with $\mathrm{NOX}^{-/-} \mathrm{PF}$ controls; results substantiated by RT-qPCR data of cortical osteocalcin and collagen 1a1 mRNA expression. Only EtOH-fed WT mice showed a significant decrease in cortical thickness. Given the significant decrease in trabecular bone loss in EtOH-fed WT mice, suggesting acceleration of endosteal bone turnover, medullary area was expected to significantly increase in parallel with significant 


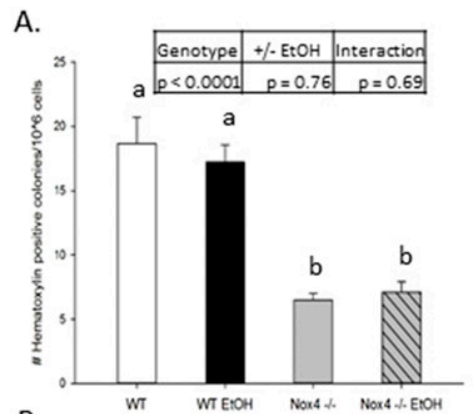

B.

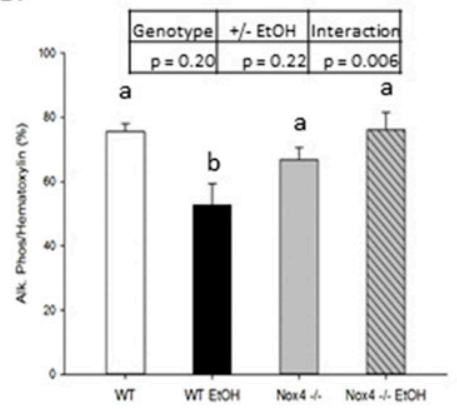

D.

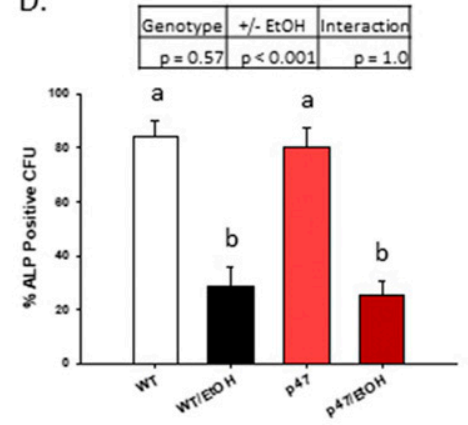

WT Pair Fed

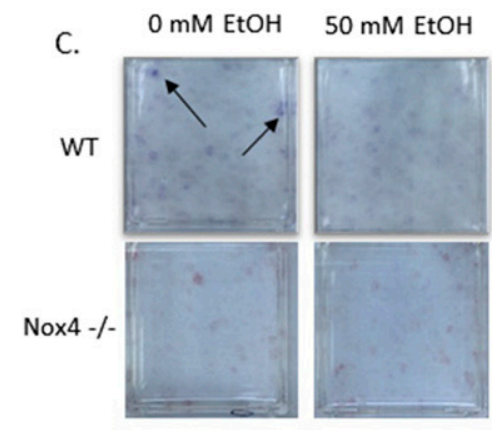

Fig. 6. $\mathrm{NOX}_{4}^{-1-} \mathrm{BM}-\mathrm{MSC}$ s from 9- to 10 -week-old male and female mice develop fewer fibroblast colonies but are protected against EtOH's suppression of AP. (A) Count of hematoxylin-positive fibroblast colonies (CFU-F) per million cells plated. (B) Percentage of hematoxylin-positive colonies that stained positive for AP. (C) Representative images of WT (top) and NOX $4^{-1-}$ (bottom) cultures treated with or without $\mathrm{EtOH}$, following staining for AP using Fast Blue (WT) or Fast Violet $\left(\mathrm{NOX} 4^{-1-}\right.$ ) salt. Arrow: representative punctate AP + CFU-F. (D) Percentage of hematoxylin-positive colonies that stained positive for AP ( $\mathrm{p} 47$ : cultures extracted from $\mathrm{p} 47^{\text {phox-1- }}$ animals lacking functional NOX2). Statistical significance was determined by two-way ANOVA; values with different letter superscripts are statistically different from each other $(P<0.05$, Tukey's multiple comparisons analysis). For each group: WT $0 \mathrm{mM}$ EtOH, $n=15$; WT $50 \mathrm{mM}$ EtOH, $n=12$; NOX $^{-l-} 0 \mathrm{mM}, n=$ 7 ; $\mathrm{NOX}^{-/-} 50 \mathrm{mM}, n=7$.

cortical thinning. However, it appeared that the slight increase in total area with EtOH in WT mice, along with measurement variability, may have obscured this observation. In summary, NOX4 deletion appears to be protective of EtOH's reduction in cortical thickness but susceptible to EtOH's reduction in overall bone and total area.

RT-qPCR analyses of specific mRNA targets in trabecular bone suggest a compartment-specific effect of NOX4 and EtOH: in contrast to cortical bone, vertebral bone (which is predominately trabecular) had suppressed collagen 1a1 expression with EtOH regardless of genotype (Fig. 2D). Differences in the regulation of bone mass in the cortical versus trabecular compartments have been reported (e.g., Alund et al., 2016, 2017). For example, Alund et al. (2017) showed that dietary antioxidant coadministration protected against trabecular bone loss but not cortical bone loss with EtOH. Similarly, transgenic mice overexpressing human catalase were protected against EtOH-induced loss of trabecular bone, although cortical bone mineral density was significantly reduced (Alund et al., 2016). One notable difference between the two compartments is the greater osteocyte population in the cortex, which contributes to bone remodeling by promoting osteoclast differentiation through RANKL expression (Sims and Martin, 2014) and secreting mineralizing factors such as DMP1 and PHEX (Bonewald, 2011). Moreover, NOX4 plays a direct role in osteoclast differentiation downstream from RANKL/RANK binding (Yang et al., 2004); therefore, global deletion of NOX4 may supersede any osteoclastic effect of increased RANKL mRNA expression in cortical bone osteocytes (Fig. 2F). This idea is supported by increases in NOX2 mRNA expression (Fig. 2E) previously shown to be associated with RANKL expression (Mercer et al., 2014). Future studies using Cre-Lox models that delete NOX4 or NOX2 specifically in early osteoblasts or mature osteocytes, or by site-specific deletion driven by the long bone-specific Prx-1 promoter (Logan et al., 2002), will help elucidate the apparent compartmental or site-specific effects related to NOX4.

The Wnt pathway, which supports bone formation, is suppressed by oxidative stress (Almeida et al., 2007a). Chen et al. (2010) showed that the Wnt pathway is a sensitive target of EtOH's effect on bone through an oxidative stress-related mechanism. In this context, Wnt pathway targets in samples of vertebral bone were probed to confirm whether the suppressive effect of EtOH was NOX4 dependent. In contrast to 
Chen et al. (2010), where administration of the dietary antioxidant $N$-acetylcysteine eliminated EtOH's suppression of Wnt-related genes, NOX4 deletion (which might also be expected to blunt the production of ROS) did not protect against suppression of the end target gene Ccnd2. In general, EtOH suppressed various steps along the canonical pathway. Thus, although NOX4 is a significant source of oxidative stress, and ROS interfere with canonical Wnt signaling (Almeida et al., 2007a; Chen et al., 2010), there appear to be other EtOH-induced oxidative stress mechanisms (e.g., mitochondrial ROS) that contribute to a ROS-related reduction in Wnt signaling independent of NOX4.

Epithelial models employing angiotensin II have provided evidence of crosstalk between NOX-induced and mitochondrial ROS (Dikalov, 2011). Deleting p22 $2^{\text {phox }}$ (the common subunit among NOX isoforms) blocks mitochondrial ROS increases by angiotensin II (Doughan et al., 2008). Conversely, blocking mitochondrial superoxide dismutase (causing an increase in mitochondrial superoxide) elevates NOX activity; together, these mechanisms amplify ROS (Dikalova et al., 2010). NOX isoforms also show interdependence. Among the NADPH oxidases, hydrogen peroxide increases NOX2 superoxide radical formation in fibroblasts (Li et al., 2001). Future studies will resolve the complex picture describing ROS generation as a function of multiple intracellular sources using mitochondria-specific inhibitors (e.g., MitoTEMPO) and cell type-specific and isoform-specific NOX knockout models.

An important finding in this study is that NOX4 deletion results in a lower capacity to form fibroblast colonies (CFU-F). It also bears noting that these in vitro results paralleled the static histomorphometry analyses, showing a smaller population of osteoblasts that, on a per area basis, produced less bone in response to $\mathrm{EtOH}$. It has been suggested that an optimal level of ROS is necessary for appropriate osteoblast differentiation (Schröder, 2015). Deletion of NOX4 may present a phenotype whereby MSCs have a diminished capacity for selfrenewal or for the early stages of differentiation. As a result, the effects of NOX4 deletion and $\mathrm{EtOH}$ on bone formation, particularly in the MSC-rich trabecular compartment, are additive.

Bone loss results from a severe imbalance of resorption and formation. NOX4 deletion protects bone from EtOH-induced mineral loss that is observable in the osteocyte-dominated cortical compartment. Although it appears a compensatory upregulation of NOX2 under NOX4 deletion is concurrent with an upregulation of RANKL in cortical bone, exactly how this effect may interact with a loss of NOX4-dependent osteoclast differentiation is not yet known. Non-bone sources of RANKL, such as the EtOH-injured liver (Araújo Júnior et al., 2016), may influence this dynamic. More observable in the NOX $4^{-/-}$model was suppression of functional osteoblast differentiation that would target the osteoblast-rich trabecular compartment, generating a lower baseline level of trabecular bone. Further studies will elucidate, via cell-specific knockouts, the NOX isoform-dependent steps in osteoblast differentiation and target the intracellular communication between osteoblasts and osteoclasts that functionally determines the balance of bone turnover in the presence of $\mathrm{EtOH}$.

\section{Authorship Contributions}

Participated in research design: Ronis, Alund, Pulliam, Watt, Mercer.
Conducted experiments: Alund, Pulliam, Watt.

Performed data analysis: Alund, Watt, Chen, Suva.

Wrote or contributed to the writing of the manuscript: Watt, Alund, Ronis, Suva.

\section{References}

Almeida M, Han L, Martin-Millan M, O'Brien CA, and Manolagas SC (2007a) Oxidative stress antagonizes Wnt signaling in osteoblast precursors by diverting $\beta$-catenin from T cell factor- to forkhead box O-mediated transcription. J Biol Chem 282:27298-27305.

Almeida M, Han L, Martin-Millan M, Plotkin LI, Stewart SA, Roberson PK, Kousteni S, O'Brien CA, Bellido T, Parfitt AM, et al. (2007b) Skeletal involution by ageassociated oxidative stress and its acceleration by loss of sex steroids. J Biol Chem 282:27285-27297.

Alund AW, Mercer KE, Pulliam CF, Suva LJ, Chen JR, Badger TM, and Ronis MJ (2017) Partial protection by dietary antioxidants against ethanol-induced osteopenia and changes in bone morphology in female mice. Alcohol Clin Exp Res 41: $46-56$

Alund AW, Mercer KE, Suva LJ, Pulliam CF, Chen JR, Badger TM, Van Remmen H, and Ronis MJJ (2016) Reactive oxygen species differentially regulate bone turnover in an age-specific manner in catalase transgenic female mice. $J$ Pharmacol Exp Ther 358:50-60.

Ambe K, Watanabe H, Takahashi S, and Nakagawa T (2014) Immunohistochemical localization of Nox1, Nox4 and Mn-SOD in mouse femur during endochondral ossification. Tissue Cell 46:433-438.

Araújo Júnior RF, Garcia VB, Leitão RF, Brito GA, Miguel Ede C, Guedes PM, and de Araújo AA (2016) Carvedilol improves inflammatory response, oxidative stress and fibrosis in the alcohol-induced liver injury in rats by regulating Kuppfer cells and hepatic stellate cells. PLoS One 11:e0148868.

Atashi F, Modarressi A, and Pepper MS (2015) The role of reactive oxygen species in mesenchymal stem cell adipogenic and osteogenic differentiation: a review. Stem Cells Dev 24:1150-1163.

Bai XC, Lu D, Liu AL, Zhang ZM, Li XM, Zou ZP, Zeng WS, Cheng BL, and Luo SQ (2005) Reactive oxygen species stimulates receptor activator of NF- $\kappa \mathrm{B}$ ligand expression in osteoblast. $J$ Biol Chem 280:17497-17506.

Bedard K and Krause KH (2007) The NOX family of ROS-generating NADPH oxidases: physiology and pathophysiology. Physiol Rev 87:245-313.

Bonewald LF (2011) The amazing osteocyte. J Bone Miner Res 26:229-238.

Bouxsein ML, Boyd SK, Christiansen BA, Guldberg RE, Jepsen KJ, and Müller R (2010) Guidelines for assessment of bone microstructure in rodents using microcomputed tomography. J Bone Miner Res 25:1468-1486.

Brandes RP, Weissmann N, and Schröder K (2014) Nox family NADPH oxidases: molecular mechanisms of activation. Free Radic Biol Med 76:208-226.

Callaci JJ, Himes R, Lauing K, Wezeman FH, and Brownson K (2009) Binge alcoholinduced bone damage is accompanied by differential expression of bone remodeling-related genes in rat vertebral bone. Calcif Tissue Int 84:474-484.

Carnesecchi S, Deffert C, Donati Y, Basset O, Hinz B, Preynat-Seauve O, Guichard C, Arbiser JL, Banfi B, Pache JC, et al. (2011) A key role for NOX4 in epithelial cell death during development of lung fibrosis. Antioxid Redox Signal 15:607-619.

Chen JR, Haley RL, Hidestrand M, Shankar K, Liu X, Lumpkin CK, Simpson PM, Badger TM, and Ronis MJJ (2006) Estradiol protects against ethanol-induced bone loss by inhibiting up-regulation of receptor activator of nuclear factor- $\kappa \mathrm{B}$ ligand in osteoblasts. J Pharmacol Exp Ther 319:1182-1190.

Chen JR, Lazarenko OP, Haley RL, Blackburn ML, Badger TM, and Ronis MJ (2009) Ethanol impairs estrogen receptor signaling resulting in accelerated activation of senescence pathways, whereas estradiol attenuates the effects of ethanol in osteoblasts. J Bone Miner Res 24:221-230.

Chen JR, Lazarenko OP, Shankar K, Blackburn ML, Badger TM, and Ronis MJ (2010) A role for ethanol-induced oxidative stress in controlling lineage commitment of mesenchymal stromal cells through inhibition of Wnt $/ \beta$-catenin signaling. $J$ Bone Miner Res 25:1117-1127.

Chen JR, Lazarenko OP, Shankar K, Blackburn ML, Lumpkin CK, Badger TM, and Ronis MJJ (2011) Inhibition of NADPH oxidases prevents chronic ethanolinduced bone loss in female rats. J Pharmacol Exp Ther 336:734-742.

Chen JR, Shankar K, Nagarajan S, Badger TM, and Ronis MJJ (2008) Protective effects of estradiol on ethanol-induced bone loss involve inhibition of reactive oxygen species generation in osteoblasts and downstream activation of the extracellular signal-regulated kinase/signal transducer and activator of transcription 3/ receptor activator of nuclear factor- $\kappa \mathrm{B}$ ligand signaling cascade. $J$ Pharmacol Exp Ther 324:50-59.

Dallas SL and Bonewald LF (2010) Dynamics of the transition from osteoblast to osteocyte. Ann N Y Acad Sci 1192:437-443.

Dempster DW, Compston JE, Drezner MK, Glorieux FH, Kanis JA, Malluche H, Meunier PJ, Ott SM, Recker RR, and Parfitt AM (2013) Standardized nomenclature, symbols, and units for bone histomorphometry: a 2012 update of the report of the ASBMR Histomorphometry Nomenclature Committee. J Bone Miner Res 28: $2-17$.

Dikalov S (2011) Cross talk between mitochondria and NADPH oxidases. Free Radic Biol Med 51:1289-1301.

Dikalova AE, Bikineyeva AT, Budzyn K, Nazarewicz RR, McCann L, Lewis W, Harrison DG, and Dikalov SI (2010) Therapeutic targeting of mitochondrial superoxide in hypertension. Circ Res 107:106-116.

Doughan AK, Harrison DG, and Dikalov SI (2008) Molecular mechanisms of angiotensin II-mediated mitochondrial dysfunction: linking mitochondrial oxidative damage and vascular endothelial dysfunction. Circ Res 102:488-496.

Duan P and Bonewald LF (2016) The role of the Wnt/ $\beta$-catenin signaling pathway in formation and maintenance of bone and teeth. Int $J$ Biochem Cell Biol 77: 23-29. 
Gaddini GW, Turner RT, Grant KA, and Iwaniec UT (2016) Alcohol: a simple nutrient with complex actions on bone in the adult skeleton. Alcohol Clin Exp Res 40: 657-671.

Goettsch C, Babelova A, Trummer O, Erben RG, Rauner M, Rammelt S, Weissmann N, Weinberger V, Benkhoff S, Kampschulte M, et al. (2013) NADPH oxidase 4 limits bone mass by promoting osteoclastogenesis. J Clin Invest 123:4731-4738.

González-Reimers E, Alvisa-Negrín J, Santolaria-Fernández F, Ros-Vilamajó R, Martín-González MC, Hernández-Betancor I, García-Valdecasas-Campelo E, and González-Díaz A (2011) Prognosis of osteopenia in chronic alcoholics. Alcohol 45:227-238.

Komori T, Yagi H, Nomura S, Yamaguchi A, Sasaki K, Deguchi K, Shimizu Y, Bronson RT, Gao YH, Inada M, et al. (1997) Targeted disruption of Cbfa1 results in a complete lack of bone formation owing to maturational arrest of osteoblasts. Cell 89:755-764.

Li WG, Miller FJ, Jr, Zhang HJ, Spitz DR, Oberley LW, and Weintraub NL (2001) $\mathrm{H}_{2} \mathrm{O}_{2}$-induced $\mathrm{O}_{2}^{-}$production by a non-phagocytic $\mathrm{NAD}(\mathrm{P}) \mathrm{H}$ oxidase causes oxidant injury. J Biol Chem 276:29251-29256.

Logan M, Martin JF, Nagy A, Lobe C, Olson EN, and Tabin CJ (2002) Expression of Cre recombinase in the developing mouse limb bud driven by a Prxl enhancer. Genesis 33:77-80.

Long $\mathrm{F}$ (2011) Building strong bones: molecular regulation of the osteoblast lineage. Nat Rev Mol Cell Biol 13:27-38.

Martin TJ and Seeman E (2008) Bone remodelling: its local regulation and the emergence of bone fragility. Best Pract Res Clin Endocrinol Metab 22:701-722.

Mercer KE, Sims CR, Yang CS, Wynne RA, Moutos C, Hogue WR, Lumpkin CK, Suva LJ, Chen JR, Badger TM, et al. (2014) Loss of functional NADPH oxidase 2 protects against alcohol-induced bone resorption in female p47phox ${ }^{-1-}$ mice. Alcohol Clin Exp Res 38:672-682.

Nyquist F, Ljunghall S, Berglund M, and Obrant K (1996) Biochemical markers of bone metabolism after short and long time ethanol withdrawal in alcoholics. Bone 19:51-54.

Orwoll ES (2003) Toward an expanded understanding of the role of the periosteum in skeletal health. J Bone Miner Res 18:949-954.

Poole KES and Compston JE (2006) Osteoporosis and its management. BMJ 333: $1251-1256$.

Rzonca SO, Suva LJ, Gaddy D, Montague DC, and Lecka-Czernik B (2004) Bone is a target for the antidiabetic compound rosiglitazone. Endocrinology 145:401-406.
Santori C, Ceccanti M, Diacinti D, Attilia ML, Toppo L, D’Erasmo E, Romagnoli E, Mascia ML, Cipriani C, Prastaro A, et al. (2008) Skeletal turnover, bone mineral density, and fractures in male chronic abusers of alcohol. $J$ Endocrinol Invest 31: $321-326$.

Schröder K (2015) NADPH oxidases in bone homeostasis and osteoporosis. Cell Mol Life Sci 72:25-38.

Shankar K, Hidestrand M, Haley R, Skinner RA, Hogue W, Jo CH, Simpson P Lumpkin CK, Jr, Aronson J, Badger TM, et al. (2006) Different molecular mechanisms underlie ethanol-induced bone loss in cycling and pregnant rats. Endocrinology 147:166-178.

Shankar K, Hidestrand M, Liu X, Chen JR, Haley R, Perrien DS, Skinner RA, Lumpkin CK, Jr, Badger TM, and Ronis MJJ (2008) Chronic ethanol consumption inhibits postlactational anabolic bone rebuilding in female rats. $J$ Bone Miner Res 23:338-349.

Sims NA and Martin TJ (2014) Coupling the activities of bone formation and resorption: a multitude of signals within the basic multicellular unit. Bonekey Rep $\mathbf{3}$ : 481.

Suva LJ, Hartman E, Dilley JD, Russell S, Akel NS, Skinner RA, Hogue WR, Budde U, Varughese KI, Kanaji T, et al. (2008) Platelet dysfunction and a high bone mass phenotype in a murine model of platelet-type von Willebrand disease. Am J Pathol 172:430-439.

Wittrant Y, Gorin Y, Mohan S, Wagner B, and Abboud-Werner SL (2009) Colonystimulating factor-1 (CSF-1) directly inhibits receptor activator of nuclear factor$\kappa \mathrm{B}$ ligand (RANKL) expression by osteoblasts. Endocrinology 150:4977-4988.

Yang CS, Mercer KE, Alund AW, Suva LJ, Badger TM, and Ronis MJ (2014) Genistein supplementation increases bone turnover but does not prevent alcoholinduced bone loss in male mice. Exp Biol Med (Maywood) 239:1380-1389.

Yang S, Zhang Y, Ries W, and Key L (2004) Expression of Nox4 in osteoclasts. J Cell Biochem 92:238-248.

Address correspondence to: Dr. Martin J. J. Ronis, Department of Pharmacology and Experimental Therapeutics, Louisiana State University Health Sciences Center, 1901 Perdido Street, New Orleans, LA 70112. E-mail: mronis@lsuhsc.edu 